\title{
EXPECTATIVAS HACIA EL CONSUMO DE ALCOHOL Y CONDUCTA DE CONSUMO EN ADOLESCENTES DE NIVEL MEDIO
}

\section{EXPECTATIONS FOR ALCOHOL CONSUMPTION AND BEHAVIOR CONSUMPTION IN MIDDLE-LEVEL ADOLESCENTS}

Jesica Guadalupe Ahumada-Cortez ${ }^{1}$, Margarita Antonia Villar-Luis², María Magdalena Alonso-Castillo³, Nora Angélica Armendáriz-García ${ }^{3}$ y Mario Enrique Gámez-Medina ${ }^{1}$

${ }^{1}$ Escuela Superior de Enfermería Mochis, Universidad Autónoma de Sinaloa, México; ${ }^{2}$ Escola de Enfermagem de Ribeirão Preto, Universidade de São Paulo, Brasil; ${ }^{3}$ Facultad de Enfermería, Universidad Autónoma de Nuevo León, México

\section{Abstract}

Objective. To determine the relationship between expectations for alcohol consumption and consumption behavior among middle-level adolescents. Methods. A cross-sectional, correlational descriptive study of 604 adolescents from 12 public middle-level schools in a municipality of Monterrey, Nuevo León, Mexico, data were collected through 2 self-administered questionnaires The Alcohol Expectations Questionnaire (AEQ), designed by Brown et al., 1980 and the Alcohol Dependence Identification Test (AUDIT), developed by WHO and adapted in Mexico by De la Fuente and Kershenobich (1992). Results. They show that $83.3 \%$ of the adolescents had their first experimental contact of alcohol between the 10 and 15 years, in the same way in the variables of study it was found that the scales of sociability and relaxation are the ones that were most related to alcohol consumption in adolescents. Conclusion. These data suggest the need to intervene early in the adolescent population, mainly informing them of the negative implications that this consumption brings to their health, thus reducing their positive expectations about the consumption of this substance.

Keywords: Alcohol, expectations, adolescents, binge drinking

\section{Resumen}

Objetivo. Determinar la relación que existe entre las expectativas hacia el consumo de alcohol y la conducta de consumo en los adolescentes de nivel medio. Método. Estudio de diseño descriptivo correlacional con corte transversal, llevado a cabo con 604 adolescentes de 12 instituciones de nivel medio de un municipio de Monterrey, Nuevo León, México, los datos se recolectaron a través de 2 cuestionarios auto-aplicables El Cuestionario de Expectativas Hacia el Alcohol (AEQ), diseñado por Brown et al. 1980 y el Test de Identificación de Desórdenes por Uso de Alcohol [AUDIT], desarrollado por la OMS y adaptado en México por De la Fuente y Kershenobich (1992). Resultados. Muestran que el 83.3\% de los adolescentes tuvo su primer contacto experimental de alcohol entre los 10 y 15 años, de la misma forma en las variables de estudio se encontró que las escalas de sociabilidad y relajación son las que más se relacionaron con el consumo de alcohol en los adolescentes. Conclusión. Estos datos sugieren la necesidad de intervenir tempranamente en población adolescente, dándoles a conocer principalmente las implicaciones negativas que este consumo trae a su salud, disminuyendo con ello las expectativas positivas que tienen sobre el consumo de esta sustancia.

Palabras clave: Alcohol, expectativas, adolescentes, consumo excesivo de alcohol. 
El consumo de alcohol es un problema de salud pública presente a nivel mundial, su uso y abuso incrementan los daños en la salud del individuo, en 2012 3.3 millones de personas murieron debido al uso nocivo del alcohol. En las Américas y en Europa se encontraron las proporciones más altas de consumo en adolescentes de 15 a 19 años (OMS, 2015). De acuerdo al ranking global realizado en el 2010 para determinar la influencia de los factores de riesgo que determinan la perdida de salud por edad, se reporta que el consumo de alcohol es uno de los principales factores de la carga de la enfermedad medidos a través de los años de vida perdidos ajustados por discapacidad, donde se presenta que en el rango de 5 a los 14 años se posiciona como el segundo factor de riesgo y pasa como primer factor cuando el rango de edad incrementa de los 15 a los 49 años (Shield \& Rehm, 2015; Whiteford et al., 2013). En México de acuerdo a la Encuesta Nacional de Consumo de Drogas, Alcohol y Tabaco (ENCODAT) los incrementos en las prevalencias de consumo de alcohol denotan una problemática que requiere de acciones preventivas inmediatas, principalmente en el consumo excesivo que se esta presentando en los adolescentes (Consejo Nacional Contra las Adicciones-Secretaría de Salud [CONADIC-SS], 2017).

El consumo de alcohol en los adolescentes de acuerdo con la evidencia localizada hasta el momento tiende a ser parecido al tipo de consumo de los adultos. Dicho consumo puede evaluarse como: sin riesgo (sensato), de riesgo (dependiente) y dañino (perjudicial), pero en los últimos años se ha destacado un patrón de consumo intermitente en los adolescentes denominado en inglés como binge drinking o consumo excesivo episódico o circunstancial, ese consumo puede resultar particularmente dañino para ciertos problemas de salud, y se define como el patrón de consumo que eleva la concentración de alcohol en sangre a .08 gramos porciento o más. En el adulto corresponde a beber 50 más bebidas en hombres y a 4 o más bebidas en mujeres por ocasión en lapso de aproximadamente 2 horas (Castañeda \& Chaves, 2013; Huang, Ho, Wang, Lo, \& Lam, 2016; National Institute on Alcohol Abuse and Alcoholism [NIAA], 2004; OPS, 2008; Parada et al., 2011; Pinsky, Sanches, Zaleski, Laranjeira, \& Caetano, 2010; Siqueira \& Smith, 2015)

Se ha establecido que en los adultos existe un tipo de consumo de alcohol que no pone en riesgo la salud del individuo, definido como sensato o sin riesgo, sin embargo en los adolescentes el tipo de consumo en esa categoría debería ser el no consumo de la sustancia, en virtud a los trastornos relacionados al comportamiento hacia el alcohol (abuso o consumo perjudicial y dependencia) y las alteraciones con efectos directos al cerebro como la intoxicación aguda, abstinencia alcohólica, delirium, trastornos psicóticos, etc (Rodríguez, Sanchiz \& Bisquerra, 2014) además a la falta de control que los expone a conductas de riesgo como accidentes de tráfico, violencia, sexo sin protección, daños neuronales, alteraciones psíquicas y de los procesos relacionados con el aprendizaje (Carrera-Fernández, Lameiras-Fernández, Rodríguez-Castro, \& Vallejo-Medina, 2013; Sánchez-Ventura \& Prevlnfad/PAPPS, 2012)

En la actualidad el consumo de alcohol es considerado una práctica socialmente aceptada, y se le reconoce como vehículo de socialización en diversos grupos sociales como en los adolescentes; es la droga legal de inicio y su consumo incrementa el riesgo de involucrarse con otro tipo de sustancias ilícitas como la marihuana, la cocaína, entre otras (Pilatti, Godoy, \& Brussino, 2012; Romer, Duckworth, Sznitman, \& Park, 2010). Dentro de los factores de riesgos presentes para el inicio de consumo de alcohol durante la adolescencia se encuentran los familiares como la falta de calidad en la relación padres-adolescentes, los conflictos parentales y padres y hermanos consumidores. En los individuales el ser hombre, la búsqueda de sensaciones, la conducta oposicionista y desafiante desarrollada tempranamente y el dejar tempranamente la escuela. Es importante resaltar que aunque existan múltiples factores de riesgo para desarrollar la conducta de consumo, el tener pares consumidores o con conducta antisocial son los factores predictores más fuertes para iniciar el consumo de alcohol y otras drogas, estos factores operan independientemente de los factores individuales y familiares (Degenhard \& Hall, 2012, Hawkins, Catalano, \& Miller, 1992; Lema et al., 2011; López \& Rodríguez-Arias, 2010; Pilatti et al., 2012; Tirado, Aguaded, \& Marín, 2009).

Las expectativas hacia el consumo de alcohol son un factor que se ha estudiado en adolescentes y se documenta que retarda o evita el inicio de consumo mediante el modelado de la familia donde se muestren las expectativas sobre los beneficios de no consumir alcohol (Mosqueda-Díaz \& Carvalho, 2011). Las expectativas positivas iniciales hacia el consumo de alcohol en el adolescente tienen un efecto directo en el uso posterior de alcohol a los 12 meses, y de la relación 
directa de la cantidad de consumo; Además que la exposición de los niños y adolescentes a un mayor consumo por parte del grupo de pares y/o padres mantienen más expectativas positivas hacia el consumo. Por sexo se ha encontrado que las mujeres perciben más peligro en el consumo de alcohol (expectativas negativas) en comparación que los hombres (Chisolm, Manganello, Kelleher \& Marshal, 2014; Jester, Wong, Cranford, Buu, Fitzgerald \& Zucker, 2015; Pilatti et al., 2012; Salamó, Gras, \& Font-Mayolas, 2010).

Como profesional de salud es importante abordar estas temáticas en poblaciones en edades tempranas como lo son los adolescentes, esto en relación a que la carga de salud por los desordenes del uso de drogas esta incrementando en la población joven, y la función del profesional de la salud pública resulta importante en la prevención, promoción y atención de la población y así mismo prevenir y/o reducir la edad de inicio de consumo de alcohol y con ello las enfermedades mentales que conlleva su consumo. Por lo anteriormente planteado se propone el siguiente objetivo de investigación: Determinar la relación entre las expectativas hacia el consumo de alcohol y la conducta de consumo en los adolescentes de nivel medio, así como también describir el tipo de consumo de alcohol en los adolescentes.

\section{MÉTODO}

El presente estudio constituye un diseño descriptivo correlacional con corte transversal (Burns \& Grove, 2015).

\section{Participantes}

La población estuvo conformada por 11,678 adolescentes masculinos y femeninos con edades entre 12 y 15 años escolarizados inscritos en 12 secundarias de un municipio del Estado de Nuevo León, México, la media de edad fue de 13.68 años ( $D E=1.01)$, con edad mínima de 12 y la máxima de 16 años; respecto al sexo la mayor proporción corresponde al sexo masculino con un 54\%. El muestreo fue probabilístico, estratificado por institución educativa y por grupo (conglomerado) con asignación proporcional al tamaño del estrato. El tamaño de la muestra se determinó con el paquete estadístico nQuery Advisor ${ }^{\circledR} 7.0$ (Elashoff, 2007) de acuerdo a Cohen. Tomando un nivel de significancia de .05 para contraste de una correlación, donde la hipótesis alternativa bilateral tiene una potencia del 90\%, cuando la correlación es de .15, considerando una tasa de no respuesta del 5\% y tomando en cuenta el tamaño de los estratos se ajustó la muestra quedando un total de 604 adolescentes de nivel medio.

\section{Instrumentos}

El Cuestionario de Expectativas Hacia el Alcohol (AEQ), fue diseñado por Brown, Goldman, Inn \& Anderson,(1980), para población adulta el cual mide las creencias positivas del consumo de alcohol y sus efectos en la conducta social y emocional alrededor de seis dimensiones: a) transforma positivamente las experiencias; b) incrementa sexualidad; c) realza el placer físico y social; d) aumenta el asertividad social; e) reduce la tensión y f) incrementa el poder y la agresión. En el 2000 este cuestionario fue adaptado para población mexicana por Mora et al., donde se obtuvo una consistencia interna de Alpha de Cronbach = .93 (Mora-Ríos, Natera, Villatoro \& Villalvazo, 2000).

La conducta de consumo de alcohol se midió con la Prueba de Identificación de Desórdenes por Uso de Alcohol [AUDIT], desarrollado por la OMS y adaptado en México por De la Fuente y Kershenobich, ha sido utilizada en población adolescente mexicana (Tegoma-Ruiz \& Cortaza-Ramírez, 2016; Villegas-Pantoja, Alonso-Castillo, Alonso-Castillo \& Guzmán, 2014) permiten determinar los patrones de consumo de alcohol de los individuos (consumo sensato, dependiente y dañino), está conformado por 10 reactivos de opción múltiple que examinan el consumo excesivo de alcohol. Los reactivos 1 a 3 indican la cantidad y frecuencia de consumo de alcohol y determinan el consumo sensato (sin riesgo), los reactivos 4 a 6 determinan el consumo dependiente (de riesgo) y del 7 al 10 el consumo dañino (perjudicial) (De la Fuente \& Kershenobich, 1992). Los resultados oscilan de 0 a 40 puntos, donde a mayor puntuación existe mayor consumo de alcohol. El consumo excesivo episódico (binge drinking) se midió a través de la pregunta: ¿Has consumido 6 o más bebidas alcohólicas en 2 horas o menos?

\section{Procedimiento}

Para el procedimiento de colecta de los datos se acondicionó un aula donde los participantes pudieran sentirse seguros y cómodos para el llenado de los instrumentos. Dicha colecta se llevó a cabo por el investigador principal y dos estudiantes de doctorado previamente capacitados. La aplicación de los instrumentos inició con la colecta de los consentimientos y asentimientos previamente firmados (por adolescentes 
y padres o tutores), posteriormente se les hizo entrega individual de los instrumentos auto-aplicables dentro de un sobre amarillo a fin de cuidar el anonimato.

\section{Consideraciones éticas}

El estudio se apegó a lo dispuesto en el Reglamento de la Ley General de Salud en Materia de Investigación para la Salud (Secretaría de Salud [SSA], 1987), en los aspectos éticos relacionados con la investigación en seres humanos, además fue aprobado por el Comité de Ética en Investigación y el Comité de Investigación de la Facultad de Enfermería de la Universidad Autónoma de Nuevo León y por la Secretaría de Educación Pública del Estado de Nuevo León, para conseguir la autorización de los padres de familia se realizaron reuniones de padres de familia para explicar el objetivo y responder cualquier duda que pudiera surgir respecto con la participación de sus hijos en la investigación.

\section{Análisis de datos}

Para el análisis de datos se utilizó el paquete estadístico para las ciencias sociales SPSS versión 20.0 para Mac OSX. Primeramente, se revisó la consistencia interna de cada una de las escalas utilizadas a través del Coeficiente de Confiabilidad Alpha de Cronbach, posteriormente se utilizó estadística descriptiva e inferencial. El análisis descriptivo de las variables continuas y categóricas se realizó a través de frecuencias, proporciones, medidas de tendencia central y de variabilidad. Para contrastar la hipótesis de normalidad en las variables continuas se aplicó la Prueba de Bondad de Ajuste de Kolmogorov-Smirnov con Corrección de Lilliefors, y se decidió el uso de pruebas no paramétricas para dar respuesta a los objetivos de investigación, a través del Coeficiente de Correlación de Spearman y el Modelo de Regresión Múltiple. Para el análisis de las variables se tomó como variable dependiente la conducta de consumo de alcohol y como variables independientes: sexo, edad, riesgo y agresividad, estados negativos, deterioro cognitivo y conductual, sociabilidad y relajación.

\section{RESULTADOS}

Al revisar la edad de primer contacto con el consumo de alcohol, se observa que el $83.3 \%$ de los adolescentes reportó que su primer contacto con el consumo de alcohol fue entre los 10 y 15 años, con una media de edad de 11.5 años. El tipo de bebidas alcohólicas de preferencia consumidas por los adolescentes son: la cerveza, que permanece como la bebida de mayor preferencia entre hombres y mujeres (51.4\%), seguida por las bebidas preparadas (29.4\%) y los vinos de mesa (9.0\%).

En la prueba Kolmogorov-Smirnov con corrección Lilliefors para las variables continuas, los resultados de las variables no presentaron una distribución normal $(p<.001)$, por lo que se rechazó la hipótesis de normalidad y se decidió el uso de estadística inferencial no paramétrica para responder a los objetivos de la investigación.

En relación al consumo de alcohol y la prevalencia de consumo alguna vez en la vida, en el último año y en el último mes. Se observa que el 70.4\% (IC95\% 67-74) de los adolescentes consumió alcohol alguna vez en la vida, en la prevalencia del último año el 53.6\% (IC95\% 50-58) consumió alcohol y el $20.5 \%$ (IC95\% 17-24) consumió en el último mes. Es importante resaltar que 280 participantes (46.4\%) se mantienen en la conducta de abstención. Por otra parte, en lo que respecta al binge drink o consumo excesivo episódico el 13.7\% (IC95\% 11-16) de los adolescentes reportaron este tipo de consumo excesivo, expresado en consumir más de 6 bebidas alcohólicas en un promedio de 2 horas por ocasión, siendo los hombres quienes presentan mayormente este tipo de consumo (62.7\%)

Al hacer la comparación del consumo excesivo episódico o binge drink con las subescalas, se encontró que los adolescentes que tenían un consumo excesivo episódico mostraron medias y medianas más altas en las subescalas de sociabilidad $(U=11295.0, p<.001)$ y relajación $(U=11969.0, p<.001)$ en comparación con aquellos que no lo tenían, además se encontró diferencias significativas en la subescala de estados negativos $(U=17369.0, p=.004)$ donde los adolescentes que no tenían un consumo excesivo mostraron medias y medianas más altas, es decir, a mayor percepción de estados negativos con el consumo de alcohol menor es el consumo.

Para describir las dimensiones que componen las expectativas hacia el consumo de alcohol por sexo, se aplicó la U de Mann-Whitney, los resultados se presenta en la Tabla 1. Los resultados muestran diferencias significativas en las dimensiones de relajación, riesgo y agresividad, y en las puntuaciones de la escala general donde los hombres presentan medias y medianas más altas en comparación con las mujeres. 
Tabla 1. Prueba de U de Mann- Whitney para la escala de expectativas hacia el alcohol por sexo

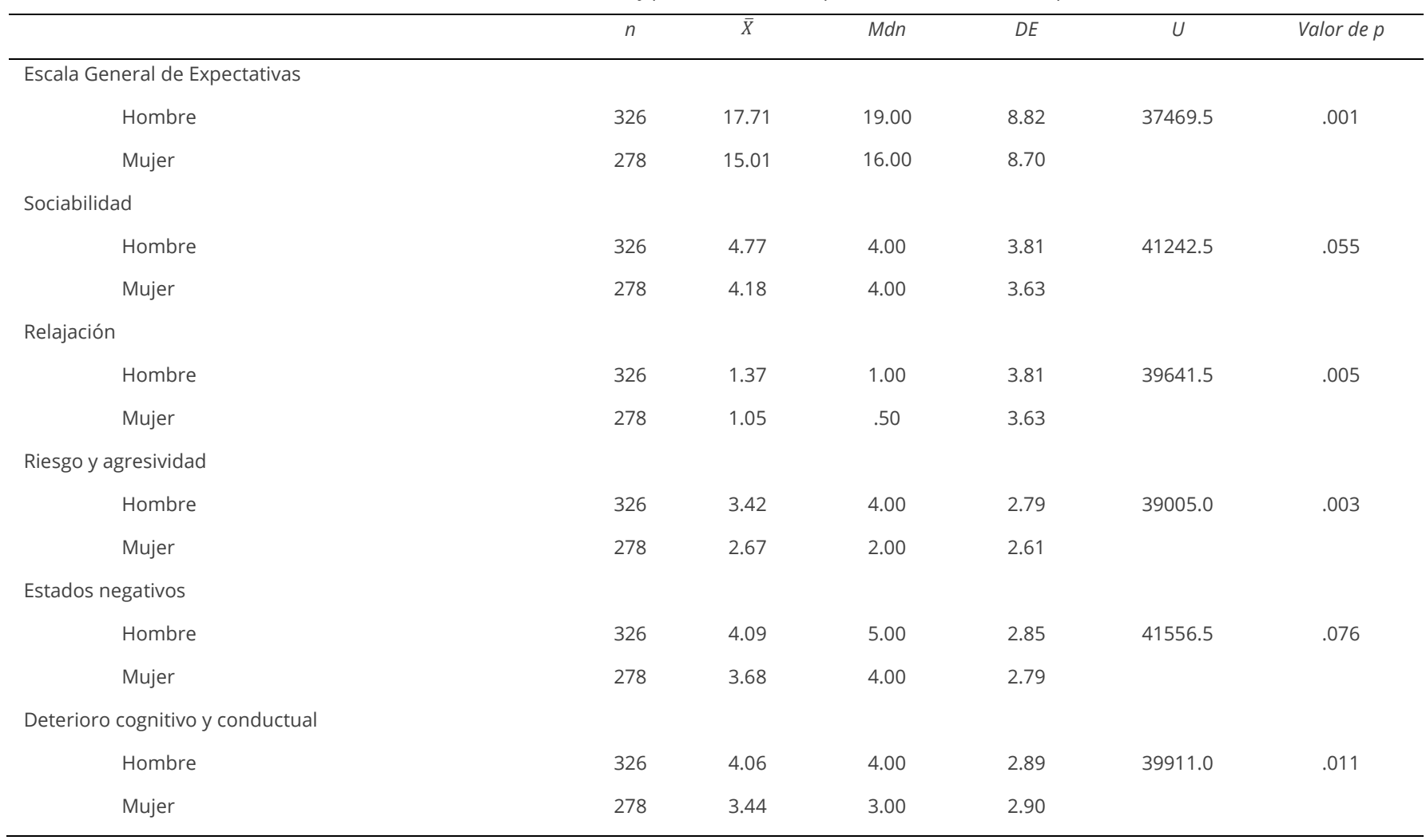

Nota: $\mathrm{n}=604$

En la Tabla 2. Se presentan los resultados del Coeficiente de Correlación de Spearman, donde se muestra que el consumo de alcohol presenta una correlación positiva y significativa con las dimensiones de relajación y sociabilidad, es decir, cuando el adolescente percibe mayor relajación al consumir alcohol, mayor es el consumo de alcohol que se presenta, de igual forma, al percibir el adolescente que al consumir alcohol es mayor la sociabilidad con sus iguales, mayor es el consumo.

Tabla 2. Coeficiente de Correlación de Spearman de Expectativas Hacia el Alcohol y Consumo de Alcohol

\begin{tabular}{|c|c|c|c|c|c|c|c|}
\hline & 1 & 2 & 3 & 4 & 5 & 6 & 7 \\
\hline 1. Escala General de Expectativas & 1 & & & & & & \\
\hline 3. Relajación & $.428^{* *}$ & $.694^{* *}$ & 1 & & & & \\
\hline 4. Riesgo y agresividad & $.710^{\star \star}$ & .041 & .048 & 1 & & & \\
\hline 5. Estados negativos & $.747 * \star$ & .017 & -.014 & $.602 * \star$ & 1 & & \\
\hline 6. Deterioro cognitivo y conductual & $.762^{\star \star}$ & .053 & .049 & $.562^{\star \star}$ & $.759 * *$ & 1 & \\
\hline 7. Consumo de alcohol & $.246^{\star \star}$ & $.380 * \star$ & $.392 \star \star$ & .061 & -.045 & .063 & 1 \\
\hline
\end{tabular}

Nota: ${ }^{*} \mathrm{p}<.01 .{ }^{*} \mathrm{p}<.05 ; \mathrm{n}=604$

En la Tabla 3 se muestran los resultados de los Coeficientes de Regresión Lineal Múltiple entre las subescalas que componen la escala de expectativas y el puntaje total del AUDIT como variable dependiente. Es importante destacar que en este modelo sólo se toman en cuentan los adolescentes que consumieron alcohol en el último año, de tal manera que permite observar el efecto de las variables sobre el consumo de alcohol en los adolescentes consumidores de alcohol. Los resultados de los modelos fueron significativos, donde las variables que más aportaron al modelo fueron las subescalas de sociabilidad y relajación. 
Tabla 3. Modelos de Regresión Lineal Múltiple por Consumo de Alcohol

\begin{tabular}{|c|c|c|c|c|c|}
\hline \multirow{2}{*}{\multicolumn{2}{|c|}{ Modelo inicial }} & \multicolumn{2}{|c|}{$\begin{array}{l}\text { Coeficientes no } \\
\text { estandarizados }\end{array}$} & \multirow{2}{*}{$\begin{array}{c}\begin{array}{c}\text { Coeficientes } \\
\text { estandarizados }\end{array} \\
\beta\end{array}$} & \multirow{2}{*}{ Valor de $\mathrm{p}$} \\
\hline & & $\mathrm{B}$ & $\mathrm{EE}$ & & \\
\hline & (Constante) & 4.388 & .809 & & .001 \\
\hline & Sociabilidad & .367 & .116 & .199 & .002 \\
\hline & Relajación & 1.097 & .312 & .224 & .001 \\
\hline & Riesgo y agresividad & .167 & .164 & .066 & .309 \\
\hline & Estados negativos & -.377 & .190 & -.151 & .048 \\
\hline & Deterioro cognitivo y conductual & .114 & .178 & .046 & .524 \\
\hline \multicolumn{6}{|c|}{$F_{(5,323)}=11.985, R^{2}=15.9 \%, p=.001$} \\
\hline \multicolumn{6}{|c|}{ Modelo backward } \\
\hline & (Constante) & 3.843 & .637 & & .001 \\
\hline & Sociabilidad & .354 & .116 & .192 & .002 \\
\hline & Relajación & 1.177 & .307 & .240 & .001 \\
\hline \multicolumn{6}{|c|}{$F_{(2,323)}=27.744, R^{2}=14.7 \%, p=.001$} \\
\hline
\end{tabular}

\section{DISCUSIÓN Y CONCLUSIONES}

En relación a los objetivos del presente estudio y las contribuciones en la práctica de los profesionales de la salud, primeramente, estos resultados permiten reconocer como se encuentra el consumo de alcohol en los adolescentes de educación media y cuales son aquellas percepciones sobre las diferentes expectativas que los motivan a iniciar o mantener el consumo de alcohol a tan corta edad, además sirven de base para la propuesta y diseño de nuevos programas preventivos. Se propone también la inclusión de estos conocimientos al currículo de formación de los profesionales de la salud.

Un dato importante de señalar es el consumo excesivo episódico de alcohol (binge drinking) que en el estudio se reporta por los adolescentes en un $13.7 \%$, este tipo de consumo es similar al que se presenta en España en el Ilamado "botellón" caracterizado por una alta ingesta en un corto periodo de tiempo, al menos cinco copas por encuentro cada fin de semana y en casos mas graves este consumo es diario (Choquet, 2010; Elzo, 2010). Este patrón de consumo según el National Institute on Alcohol Abuse and Alcoholism (NIAA, 2015) lo define como un típico consumo de cuatro y cinco copas (mujer y hombre respectivamente) lo que puede alcanzar a tener concentraciones de $0.08 \mathrm{gr} / \mathrm{dl}$ de alcohol en sangre por lo que es peligroso y presenta con rapidez disrupciones en el estado de ánimo y el comportamiento con claros problemas de coordinación, además de daños cardíacos, hepáticos, pancreáticos y del sistema inmunológico a corto plazo. En México, la última Encuesta Nacional (ENCODAT, 2017), reporta que este mismo consumo excesivo en el último mes en población adolescente de 12 a 17 años se duplicó del 2011 al 2016 pasando de 4.3\% al $8.3 \%$ respectivamente.

Respecto al consumo de alcohol (excesivo episódico) por sexo se observan diferencias significativas donde los hombres presentan un mayor consumo. Estos datos coinciden con los reportes de la ENCODAT (2017) donde muestran que el consumo excesivo en hombres es mayor que en las mujeres con un $8.9 \%$ y un $7.7 \%$ respectivamente. Además coinciden con datos encontrados por García, Calvo, Carbonell y Giralt (2017) y Mcbride, Barrett, Moore y Schonfeld (2014). Hallazgos que pueden explicarse probablemente a que los adolescentes varones asocian la conducta del consumo de alcohol excesivo como sinónimo de fortaleza y hombría, además que a nivel contextual, es mas aceptable el consumo de alcohol de adolescentes varones que en mujeres (Alonso-Castillo et al., 2009). En contraparte las normas sociales en mujeres (feminidad) juegan un rol protector para este consumo (SánchezLópez, Rivas-Diez \& Cuéllar-Flores, 2013). 
En lo que respecta a las expectativas hacia el consumo de alcohol se encontró que a mayores expectativas positivas mayor es el consumo de alcohol en los adolescentes, estos resultados coinciden con lo encontrado por otros autores donde mencionan que los adolescentes con consumo problemático de alcohol presentan puntajes más altos en las expectativas positivas hacia el alcohol en comparación a los consumidores sensatos (Grazioli, Lewis, Garberson, Fossos-Wong, Lee \& Larimer, 2015; McBride et al., 2014; Pedersen, Mayers, Browne \& Norman, 2014; Vilenne \& Quertemont, 2015).

Por subescalas se documentó que las dimensiones de sociabilidad y relajación presentaron medias más altas en relación con el consumo dañino. Datos similares encontraron Jester et al., (2015) donde mencionan que las expectativas de socialización y relajación predicen un consumo intenso de alcohol. Esto se puede explicar a través de los conocimientos previos que tienen los adolescentes acerca de la sustancia, las experiencias que mantiene con ellas y las expectativas que suponen respecto a su consumo lo que permiten conformar las actitudes frente al acto de consumir. Estos resultados concuerdan con los hallazgos reportados por Dunne et al., 2015 y Pilatti et al., 2012, donde señalan que un puntaje mayor en la escala de sociabilidad corresponde a presentar experiencias directas de consumo, con una mayor frecuencia y en mayor cantidad, de la misma forma cuando incrementa la percepción de relajación al consumir alcohol se correlaciona positiva y significativamente con la frecuencia de consumo de alcohol (Newton, Barret, Swaffield \& Teesson, 2014; Pilatti, Cupani \& Pautassi, 2015). Estos resultados se pueden explicar a través del Modelo de Promoción de la Salud (Pender et al., 2010), este modelo es ampliamente utilizado como estrategia para el desarrollo y explicación de estilos de vida saludables (Giraldo, Toro, Macías, Valencia \& Palacios, 2010), donde se indica que el mejor predictor de la conducta es la frecuencia de la misma o de un comportamiento similar en el pasado. Este modelo propone que la conducta previa tiene efectos directos e indirectos sobre la probabilidad de participar en comportamientos similares en el futuro. Es por ello que el profesional de la salud tiene un papel muy importante en la ayuda y orientación a los individuos, donde coadyuve a construir y dar forma a una historia o expectativa a la conducta positiva o conducta promotora de la salud, en este caso la conducta de abstinencia, centrándose en los beneficios de esta conducta, además de orientar al adolescente en el cómo poder superar los obstáculos que existen para la realización de la conducta y para generar altos niveles de eficacia y afecto positivo de la experiencia a través del desempeño exitoso y la retroalimentación positiva (Pender et al., 2010).

Es importante resaltar que en el adolescente se busca la abstinencia o el retardar el inicio del consumo de alcohol el mayor tiempo posible, no sólo por las implicaciones legales que competen el consumo en menores de edad, sino en los daños tantos físicos y cognitivos a los que se enfrentan con este tipo de consumo, ya que los expone a conductas sexuales de riesgo y a una dependencia temprana a la sustancia (García et al., 2017). Por otra parte al ser un grupo poblacional en riesgo y al no poderse evitar el consumo, se deben implementar programas orientados a la concientización de la evaluación de los riesgos a la salud y la importancia del consumo responsable para así disminuir los riesgos asociados a este consumo.

De acuerdo al tipo de estudio las limitaciones que presentan son: que los análisis no pueden establecer una causa - efecto, sólo muestra la relación y la explicación de las variables de estudio, por lo que los resultados pueden extrapolarse (no generalizar) a poblaciones de similares características sociodemográficas, otra limitación es no haber incluido en el análisis de los datos presentados la edad de inicio del consumo de alcohol de los adolescentes participantes.

\section{REFERENCIAS}

Alonso-Castillo, M. M., Álvarez-Bermúdez, J., López-García, K. S., Rodríguez-Aguilar, L., Alonso-Castillo, M. T. J., Angélica-Armendáriz, N. (2009). Factores de riesgo personales, psicosociales y consumo de alcohol en mujeres adultas. Investigación en Enfermería: Imagen y Desarrollo, 11, (1), 97-114.

Brown, S. A., Goldman, M. S., Inn, A., \& Anderson, L. R. (1980). Expectations of reinforcement from alcohol their domain and relation to drinking patterns. Journal Consult Clinic Psychologic, 48, 419-426.

Burns, N., \& Grove, S. K. (2015). The Practice of Nursing Research: Conduct, critique, and utilization. (Elsevier/Saunders, Ed.) (5th ed.). St. Louis, Missouri.

Carrera-Fernández, M.-V., Lameiras-Fernández, M., Rodríguez-Castro, Y., \& Vallejo-Medina, P. (2013). Bullying Among Spanish Secondary Education Students: The Role of Gender Traits, Sexism, and 
Homophobia. Journal of Interpersonal Violence, 28(14), 2915-2940. https://doi.org/10.1177/0886260513488695

Castañeda, J. A., \& Chaves, L. (2013). The Relationship between Personality Styles and Rate of Alcoholic Consumption. Pensamiento Psicológico, 11(1), 55-69.

Consejo Nacional Contra las Adicciones-Secretaría de Salud [CONADIC-SS] Encuesta Nacional de Consumo de Drogas, Alcohol y Tabaco 20162017: Reporte de Alcohol (2017). Instituto Nacional de Salud Pública. Cuernavaca, Morelos. https://drive.google.com/file/d/1rMIKaWy34GR51sEnBK2u2q_BDK9LA0e/view

Chisolm, D. J., Manganello, J. A., Kelleher, K. J., \& Marshal, M. P. (2014). Health literacy, alcohol expectancies, and alcohol use behaviors in teens. Patient Education and Counseling, 97(2), 291-296. https://doi.org/10.1016/j.pec.2014.07.019

Choquet, M. (2010). Los jóvenes europeos y el alcohol: nuevos resultados. En Elzo, J. Hablemos de alcohol: por un nuevo paradigma en el beber adolescente: 130-170 Eds. Madrid Entinema.

Dagenhardt, L. \& Hall, W. (2012). Extent of illicit drug use and dependence, and their contribution to the global burden of disease. Lancet, 379, 55-70.

De la Fuente, J. R., \& Kershenobich, D. (1992). El alcoholismo como problema médico. Revista Facultad de Medicina UNAM, 35(2), 47-51.

Dunne, E. M., Katz, E. C., Baron, R., Kenny, D., Benotsch, E., Snipes, D., ... MacKinnon, D. (2015). Alcohol Outcome Expectancies and Regrettable Drinking-Related Social Behaviors. Alcohol and Alcoholism (Oxford, Oxfordshire), 50(4), 393-8. https://doi.org/10.1093/alcalc/agv026

Elashoff, J. D. (2007). nQuery Advisor (versión 7.0) [Software de computación]. Los Ángeles, CA, Estados Unidos: Statistical Solutions Ltd.

Elzo, J. (2010). ¿Hay un modelo mediterraneo de consumo de alcohol? En Elzo, J(Ed). Hablemos de alcohol: 30-70. Eds Madrid: Entinema.

García, C., Calvo, F., Carbonell, X., \& Giralt, C. (2017). Consumo intensivo de alcohol y conductas sexuales de riesgo en población universitaria. Salud y Drogas, 17(1), 63-71.

Giraldo, O. A., Toro, R. M. Y., Macías, L. A. M., Valencia, G. C. A., \& Palacio, R. S. (2010). La promoción de la salud como estrategia para el fomento de estilos de vida saludables. Revista hacia la promoción de la salud, 15(1), 128-143.

Grazioli, V. S., Lewis, M. A., Garberson, L. A., Fossos-Wong, N., Lee, C. M., \& Larimer, M. E. (2015). Alcohol expectancies and alcohol outcomes: effects of the use of protective behavioral strategies. Journal of Studies on Alcohol and Drugs, 76(3), 452-458. http://doi.org/10.15288/jsad.2015.76.452

Hawkins, J. D., Catalano, R. F., \& Miller, J. Y. (1992). Risk and protective factors for alcohol and other drug problems in adolescence and early adulthood: Implications for substance abuse prevention. Psychological Bulletin, 112 (1), 64-105.
Huang, R., Ho, S. Y., Wang, M. P., Lo, W. S., \& Lam, T. H. (2016). Reported alcohol drinking and mental health problems in Hong Kong Chinese adolescents. Drug and Alcohol Dependence, 164, 47-54. https://doi.org/10.1016/j.drugalcdep.2016.04.028

Jester, J. M., Wong, M. M., Cranford, J. A., Buu, A., Fitzgerald, H. E., \& Zucker, R. A. (2015). Alcohol expectancies in childhood: Change with the onset of drinking and ability to predict adolescent drunkenness and binge drinking. Addiction, 110(1), 71-79. https://doi.org/10.1111/add.12704

López, L. S., \& Rodríguez-Arias, P. J. (2010). Factores de riesgo y de protección en el consumo de drogas en adolescentes y diferencias según edad y sexo. Psicothema, 22(4), 568-573.

McBride, N. M., Barret, B., Moore, K. A., \& Schonfeld, L. (2014). The role of positive alcohol expectancies in underage drinking among college students. Journal of American College Health, 62(6), 370-379. http://doi.org/10.1080/07448481.2014.907297

Mora-Ríos, J., Natera, G., Villatoro, J., \& Villalvazo, R.(2000). Validez factorial del cuestionario de expectativas hacia el alcohol (AEQ) en estudiantes universitarios. Psicología Conductual, 8(2), 319-328.

Mosqueda-Díaz, A., \& Carvalho, F. (2011). Factores protectores y de riesgo familiar relacionados al fenómeno de drogas, presentes en familias de adolescentes tempranos de Valparaíso, Chile. Revista LatinoAmericana Enfermagem, 19: 789-795.

National Institute on Alcohol Abuse and Alcoholism [NIAA](2004). Featuring information from the national institute on alcohol abuse and alcoholism. https://pubs.niaaa.nih.gov/publications/Newsletter/winter2004/Ne wsletter_Number3.pdf

National Institute on Alcohol Abuse and Alcoholism [NIAA] (2015). Alcohol use disorder. Consultado Agosto de 2015. En http://niaa.nih.gov/alcohol-health/overview-alcoholconsumption/alcohol-use-disorders.

Newton, N. C., Barrett, E. L., Swaffield, L., \& Teesson, M. (2014). Risky cognitions associated with adolescent alcohol misuse: Moral disengagement, alcohol expectancies and perceived self-regulatory efficacy. Addictive Behaviors, 39(1), 165-172. http://doi.org/10.1016/j.addbeh.2013.09.030

Organización Mundial de la Salud (2015). Informe mundial de la OMS destaca los impactos negativos del alcohol en la salud. Disponible en http://www.paho.org/uru/index.php?option=com_content\&view=a rticle\&id=841: informe-mundial-de-la-oms-destaca-los-impactosnegativos-del-alcohol-en-la-salud-\&ltemid=340

Organización Panamericana de la Salud (2008). Alcohol y atención primaria de la salud. Informaciones clínicas básicas para la identificación y el manejo de riesgos y problemas. Disponible en http://www.who.int/substance_abuse/publications/alcohol_atenci ón_primaria.pdf

Parada, M., Corral, M., Caamaño-Isorna, F., Mota, N., Crego, A., Rodríguez, S., \& Cadaveira, F. (2011). Definición del concepto de consumo intensivo de alcohol adolescente(bimge drinking). Addicciones, 23(1), 53-63. 
Pender, N., Murdaugh, C., \& Parsons, M. (2010). Health Promotion in Nursing Practice (6 $6^{\text {th }}$ ed). Prentice Hal.

Pedersen, E. R., Myers, U. S., Browne, K. C., \& Norman, S. B. (2014). The role of alcohol expectancies in drinking behavior among women with alcohol use disorder and comorbid posttraumatic stress disorder. J Psychoactive Drugs., 46(3), 178-187. http://doi.org/10.1080/02791072.2014.917750

Pilatti, A., Cupani, M., \& Pautassi, R. M. (2015). Personality and alcohol expectancies discriminate alcohol consumption patterns in female college students. Alcohol and Alcoholism, 50(4), 385-392. http://doi.org/10.1093/alcalc/agv025

Pilatti, A., Godoy, J. C., \& Brussino, S. A. (2012). Análisis de factores que influyen sobre el uso de alcohol de niños: Un path análisis prospectivo. Health and Addictions, 12(2), 155-192.

Pinsky, I., Sanches, M., Zaleski, M., Laranjeira, R., \& Caetano, R. (2010). Patterns of alcohol use among Brazilian adolescents. Rev Bras Psiquiatr, 32(3), 242-249.

Rodríguez, G. F. D., Sanchiz, R. M. L., \& Bisquerra, A. R. (2014). Consumo de alcohol en la adolescencia. Consideraciones médicas y orientaciones educativas. Salud mental, 37(3), 255-260.

Romer, D., Duckworth, A. L., Sznitman, S., \& Park, S. (2010). Can Adolescents Learn Self-control? Delay of Gratification in the Development of Control over Risk Taking. National Institutes of Health- PA, 11(3), 319-330. https://doi.org/10.1007/s11121-0100171-8.

Salamó, A. A., Gras, P. M., \& Font-Mayolas, S. (2010). Patrones de consumo de alcohol en la adolescencia. Psicothema, 22(2), 189-195.

Sánchez-López, M. P., Rivas-Diez, R., \& Cuéllar-Flores, I. (2013). Masculinity and femininity as predictors of tobacco and alcohol consumption in spanish university students. Health and Addictions, 13(1), 15-22.

Sánchez-Ventura, J. G., \& Previnfad/PAPPS, I. y A. (2012). Prevención del consumo de alcohol en la adolescencia. Rev Pediatr Aten Primaria.

Secretaría de Salubridad y Asistencia. (1987). Reglamento de la Ley General de Salud en Materia de Investigación para la Salud. México. Disponible en

http://www.diputados.gob.mx/LeyesBiblio/regley/Reg_LGS_MIS_or ig_06ene87.pdf

Shield, K. D., \& Rehm, J. (2015). Global risk factor rankings: the importance of age-based health loss inequities caused by alcohol and other risk factors. BMC Research Notes, 8(1), 231. https://doi.org/10.1186/s13104-015-1207-8

Siqueira, L., \& Smith, V. C. (2015). Binge Drinking. PEDIATRICS, 136(3), e718e726. https://doi.org/10.1542/peds.2015-2337

Tegoma-Ruiz, V. M. \& Cortaza-Ramírez, L. (2016). Prevalencia del consumo de alcohol en adolescentes de una secundaria de Coatzacoalcos, Veracruz. Enfermería Universitaria, 13(4), 239-245.

Tirado, M. R., Aguaded, G. J., \& Marín, G. I. (2009). Factores de protección y de riesgo del consumo de alcohol en alumnos de la universidad de Huelva. Health and Addictions, 9(2), 165-184.
Vilenne, A., \& Quertemont, E. (2015). Explicit and implicit positive alcohol expectancies in problem and non-problem drinkers: Differences across age groups from young adolescence to adulthood. Frontiers in Psychology, 6(NOV), 1-9. http://doi.org/10.3389/fpsyg.2015.01773

Villegas-Pantoja, M. Á., Alonso-Castillo, M. M., Alonso-Castillo, B. A., \& Guzmán Facundo, F. R. (2014). Eventos estresantes y la relación con el consumo de alcohol y tabaco en adolescentes. Ciencia $Y$ Enfermería, 20(1), 35-46. http://doi.org/10.4067/S071795532014000100004

Whiteford, H. A., Degenhardt, L., Rehm, J., Baxter, A. J., Ferrari, A. J., Erskine, H. E., ... Vos, T. (2013). Global burden of disease attributable to mental and substance use disorders: findings from the Global Burden of Disease Study 2010. The Lancet, 382(9904), 1575-1586. https://doi.org/10.1016/S0140-6736(13)61611-6 